\title{
Tackling the phylogenetic conundrum of Hydroidolina (Cnidaria: Medusozoa: Hydrozoa) by assessing competing tree topologies with targeted high-throughput sequencing
}

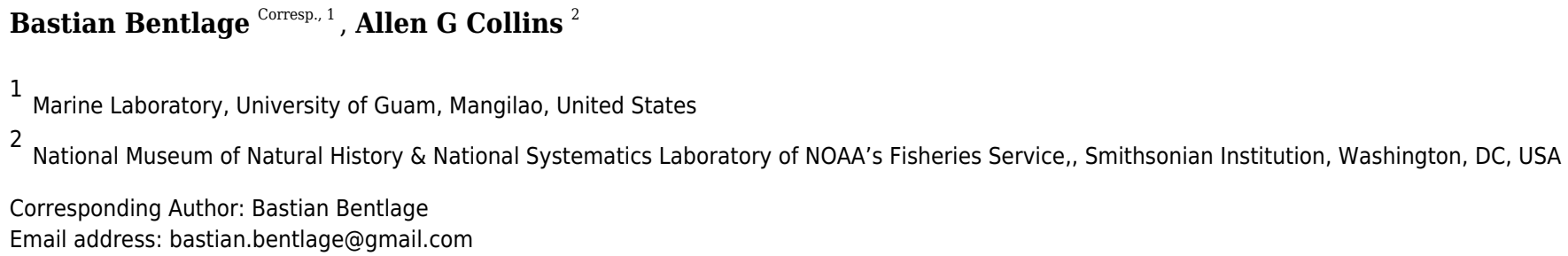

Higher-level relationships of the Hydrozoan subclass Hydroidolina, which encompasses the vast majority of medusozoan cnidarian species diversity, have been elusive to confidently infer. The most widely adopted phylogenetic framework for Hydriodolina based on ribosomal RNA data received low support for several higher level relationships. To address this issue, we developed a set of RNA baits to target more than a hundred loci from the genomes of a broad taxonomic sample of Hydroidolina for high-throughput sequencing. Using these data, we inferred the relationships of Hydroidolina using maximum likelihood and Bayesian approaches. Both inference methods yielded well-supported phylogenetic hypotheses that largely agree with each other. Using maximum likelihood and Baysian hypothesis testing frameworks, we found that several alternate topological hypotheses proposed previously may be rejected in light of the genomic data generated for this study. Both the maximum likelihood and Bayesian topologies inferred herein consistently score well across testing frameworks, suggesting that their consensus represents the most likely phylogenetic hypothesis of Hydroidolina. This phylogenetic framework places Aplanulata as sister lineage to the remainder of Hydroidolina. This is a strong deviation from previous phylogenetic analyses that placed Capitata or Siphonophorae as sister group to the remainder of Hydroidolina. Considering that Aplanulata represents a lineage comprised of species that for the most part possess a life cycle involving a solitary polyp and freeswimming medusa stage, the phylogenetic hypotheses presented herein have potentially large implications for clarifying the evolution of life cycles, coloniality, and the division of labor in Hydrozoa as taxon sampling for phylogenetic analyses becomes more complete. 
1 Tackling the phylogenetic conundrum of Hydroidolina (Cnidaria: Medusozoa: Hydrozoa)

2 by assessing competing tree topologies with targeted high-throughput sequencing.

3

4 Bastian Bentlage ${ }^{1, *}$, Allen G. Collins ${ }^{2}$

5

6 1Marine Laboratory, University of Guam, 303 University Dr., Mangilao, GU 96913, USA

$7 \quad{ }^{2}$ National Museum of Natural History \& National Systematics Laboratory of NOAA's Fisheries

8 Service, Smithsonian Institution, Washington DC, USA

9

$10 *$ Correspondence: bentlageb@triton.uog.edu 


\section{Abstract}

12 Higher-level relationships of the Hydrozoan subclass Hydroidolina, which encompasses the vast

13 majority of medusozoan cnidarian species diversity, have been elusive to confidently infer. The

14 most widely adopted phylogenetic framework for Hydriodolina based on ribosomal RNA data

15 received low support for several higher level relationships. To address this issue, we developed a

16 set of RNA baits to target more than a hundred loci from the genomes of a broad taxonomic

17 sample of Hydroidolina for high-throughput sequencing. Using these data, we inferred the

18 relationships of Hydroidolina using maximum likelihood and Bayesian approaches. Both

19 inference methods yielded well-supported phylogenetic hypotheses that largely agree with each

20 other. Using maximum likelihood and Baysian hypothesis testing frameworks, we found that

21 several alternate topological hypotheses proposed previously may be rejected in light of the

22 genomic data generated for this study. Both the maximum likelihood and Bayesian topologies

23 inferred herein consistently score well across testing frameworks, suggesting that their consensus

24 represents the most likely phylogenetic hypothesis of Hydroidolina. This phylogenetic

25 framework places Aplanulata as sister lineage to the remainder of Hydroidolina. This is a strong

26 deviation from previous phylogenetic analyses that placed Capitata or Siphonophorae as sister

27 group to the remainder of Hydroidolina. Considering that Aplanulata represents a lineage

28 comprised of species that for the most part possess a life cycle involving a solitary polyp and

29 free-swimming medusa stage, the phylogenetic hypotheses presented herein have potentially

30 large implications for clarifying the evolution of life cycles, coloniality, and the division of labor

31 in Hydrozoa as taxon sampling for phylogenetic analyses becomes more complete.

\section{Introduction}


34 While the fossil record of medusozoan cnidarians is scant, the origin of the group has been

35 inferred to lie near the end of the Ediacaran, approximately 550-580 million years ago (Han et

36 al., 2016). Plausible crown-group hydrozoans have been described from some 500 million year

37 old Cambrian deposits (Cartwright et al., 2007), suggesting an ancient origin of extant

38 hydrozoans likely dating back to the period of rapid diversification of metazoan life during

39 which all major modern animal phyla emerged (Valentine et al., 1999; Erwin, 2020).

40 Hydrozoans are of particular interest in the study of the evolution of development, as their

41 radiation gave rise to diverse life cycle strategies, diverse forms of coloniality and the division of

42 labor (Cartwright \& Nawrocki, 2010; Bentlage et al., 2018; Cartwright et al., 2020). This

43 diversity is concentrated in the hydrozoan subclass Hydroidolina, the medusozoan clade that

44 contains the vast majority of the 3,800 nominal hydrozoan species (Daly et al., 2007; Schuchert,

45 2020). Elucidating the evolutionary history and patterns of complex character evolution in

46 Hydroidolina requires a solid understanding of the phylogenetic history of the group (e.g.,

47 Cartwright et al., 2020).

48 However, the goal of inferring the deep phylogeny of Hydroidolina has been elusive,

49 possibly as a result of the early origin and likely rapid diversification of the group. The most

comprehensive phylogenetic hypothesis (Cartwright \& Nawrocki, 2010) of higher-level

51 relationships within Hydroidolina was inferred using ribosomal DNA (rDNA) from a broad

52 taxonomic sample. While shallow nodes were well resolved with high confidence, higher-level

53 relationships generally received weak support. In particular, Leptothecata, Siphonophorae,

54 Capitata, and Aplanulata were inferred to be monophyletic groups while Filifera was

55 polyphyletic (Cartwright et al., 2008; Cartwright \& Nawrocki, 2010). Previously, the latter three

56 taxa were united under the name Anthoathecata but their non-monophyly had been demonstrated 
57 earlier (Collins et al., 2006). Whole mitochondrial genomes have been employed previously to

58 address this issue and to reconstruct deep nodes within the phylogeny of Hydroidolina (Kayal et

59 al., 2015). While this approach led to a well-resolved and highly supported phylogenetic

60 hypothesis, several nodes of the resulting tree topology are at odds with rDNA-based

61 phylogenies (Cartwright et al. 2008; Cartwright \& Nawrocki, 2010) and recent phylogenomics-

62 based hypotheses (Kayal et al. 2018).

63 Advances in understanding of medusozoan, and more broadly cnidarian relationships, were

64 made by employing phylogenomic datasets derived from whole-transcriptome and genome-

65 sequencing efforts (Zapata et al., 2015; Kayal et al., 2018). While these efforts provided answers

66 to several long-standing questions of cnidarian evolutionary history, taxon sampling was

67 insufficient for rigorous evaluation of hydrozoan relationships. We used the coding sequences

68 generated by these phylogenomic studies as a backbone for targeted high-throughput sequencing,

69 producing a multi-locus dataset to infer the phylogeny of Hydroidolina. For this purpose, we

70 developed a set of custom baits to enrich target loci from a representative sample of

71 hydroidolinan hydrozoans. This work provides a new framework for the phylogeny of

72 Hydroidolina that will enable further phylogenetic comparative studies of character evolution in

73 Hydrozoa. To evaluate multiple competing topological hypotheses of hydroidolinan

74 relationships, both likelihood and Bayesian statistical frameworks (cf. Sober, 2008) were

75 employed to discriminate between alternate tree topologies and evaluate the strength of evidence

76 supporting prior phylogenetic hypotheses of Hydroidolina as well as those phylogenies inferred

77 from the multi-locus dataset presented here.

\section{Materials and Methods}


80

81

82

83

84

85

86

87

88

89

90

91

92

93

94

95

96

97

98

99

100

101

102

\section{Bait design for targeted sequencing}

Biotinylated RNA baits of $120 \mathrm{bp}$ length were designed using 355 orthogroup partitions (loci) from the phylogenomic OF-PTP_75tx matrix (Kayal et al., 2018). This dataset contains the coding sequences from a broad taxonomic sample of cnidarians, including anthozoans, medusozoans, and endocnidozoans. Due to their large sequence divergences, endocnidozoans were excluded from the dataset used for RNA bait design. Nucleotide sequences were aligned per locus guided by their predicted amino acid translations using the Geneious aligner (version 9; Biomatters, Auckland, New Zealand). Several sequences were flagged after manual audit of alignments due to their apparent divergence and identified as non-homologous sequences after BLAST searches against NCBI's GenBank. Following curation and removal of sequences shorter than $80 \mathrm{bp}$, putative repetitive elements in the remaining 5,523 sequences were softmasked using the cross_match search option against the Cnidaria repeats database in RepeatMasker (version 4.06; Smit et al., 2013-2015).

Next, sequence stretches of 10 or fewer ambiguous nucleotides $(\mathrm{N})$ were replaced with thymine $(\mathrm{T})$ repeats to allow bait design across short regions of undetermined sequences. Similarly, short sequences less than $120 \mathrm{bp}$ were padded with T repeats to allow for $120 \mathrm{bp}$ long baits to be mapped against reference sequences. Baits were designed at approximately $2.5 \mathrm{x}$ tiling density with some 50bp spacing between the start of neighboring baits, yielding 38,102 candidate baits. Each candidate bait was verified against six cnidarian genome assemblies (Table 1) using BLAST searches to evaluate the bait's fit to the reference. Hits with a length greater than $45 \mathrm{bp}$ in length and identity greater than $75 \%$ were retained for further analysis. Melting temperatures (Tm; defined as the temperature at which $50 \%$ of molecules hybridize) were estimated for each BLAST hit assuming standard Mybaits (Mycroarray, Arbor Biosciences, Ann 
103 Arbor, MI, USA) hybridization conditions. Based on the distribution of inferred Tms, baits of

104 moderate or higher specificity were retained. That is, candidate baits with at most 10 BLAST hits

105 between $62.5^{\circ} \mathrm{C}$ to $65^{\circ} \mathrm{C}$, two BLAST hits above $65^{\circ} \mathrm{C}$, and fewer than 2 baits on each flanking

106 region were retained, yielding a total of 37,546 baits (Supplementary File 1).

107

108 Target capture and sequencing

109 Genomic DNA was extracted from ethanol-preserved tissue samples (Table 2) using a standard

110 organic phenol-chloroform extraction protocol (Green \& Sambrook, 2012). Extracted DNA was

111 quantified with a Qubit 4 fluorometer (Thermo Fisher Scientific, Waltham, MA, USA). 50 $\mu 1$ of

112 DNA in TE buffer were chilled to $4^{\circ} \mathrm{C}$ in the water bath of a Q800 Sonicator (Qsonica, Newton,

113 CT, USA) and acoustically sheared for nine minutes using an amplitude of 25 with sonication

114 pulses of $15 \mathrm{~s}$ on/15s off. Illumina sequencing libraries were constructed from sheared DNA

115 samples using an NEBNext Ultra II DNA library preparation kit (New England Biolabs, Ipswich,

116 MA, USA) with dual indexes for multiplexing following the manufacturer's protocol. After

117 library amplification and magnetic bead purification using Ampure beads (Promega, Madison,

118 WI, USA), amplicons longer or shorter than approximately $200 \mathrm{bp}$ were removed using a

119 BluePippin size select gel eletrophoresis system (Sage Science, Beverly, MA, USA).

120 Concentrations of size-selected libraries were equilibrated, followed by pooling of libraries three

121 to four samples deep for target enrichment.

122 Hybridization of RNA baits to pooled libraries followed the Mybaits version 3.02 protocol

123 (Mycroarray, Arbor Biosciences, Ann Arbor, MI, USA) with the following modifications. After

124 initial denaturation and blocking with Illumina adapter-specific oligonucleotides, RNA baits

125 were allowed to hybridize for 19 hours at $65^{\circ} \mathrm{C}, 19$ hours at $60^{\circ} \mathrm{C}$, and 10 hours at $55^{\circ} \mathrm{C}$. We used 
126 this touchdown procedure on the newly developed and untested bait set in an effort to increase

127 on-target specificity while allowing for sensitivity of reactions. Following hybridization,

128 biotinylated baits were bound to streptavidin-coated magnetic beads (Dynabeads MyOne

129 Streptavidin; Thermo Fisher Scientific, Waltham, MA, USA), followed by stringent washing to

130 remove unbound DNA library molecules. Captured libraries were amplified while bound to

131 beads using KAPA HiFi DNA polymerase and HotStart ReadyMix (Roche, Basel, Switzerland)

132 following the manufacturers protocol. The annealing temperature during the 15 amplification

133 cycles was $60^{\circ} \mathrm{C}$. PCR reactions were cleaned using Ampure magentic beads (Thermo Fisher

134 Scientific, Waltham, MA, USA), washing twice with $80 \%$ ethanol. DNA concentrations were

135 quantified fluorometrically, followed by equilibration of target-enrichment pools to equimolar

136 concentrations. All reactions were pooled and the size range of the pool selected for an average

137 length of 450bp using the BluePippin size select system (Sage Science, Beverly, MA, USA).

138 Following quantification using qPCR, 300bp paired-end reads were generated on the Illumina

139 MiSeq platform with the v3 reagent kit (Illumina, San Diego, CA, USA).

140

141 Sequence assembly, alignment, and gene tree discordance

142 Sequencing adapters were removed from paired-end sequencing reads using Trimmomatic

143 (version 0.22; Bolger et al., 2014). Reads were trimmed further using a sliding window of size

144 four, with an average quality of 15 or greater required for bases within the window to be

145 retained. Quality trimmed reads shorter than 75bp were discarded. The HybPiper pipeline

146 (version 1.2; Johnson et al., 2016) was used to identify target sequences from enriched

147 sequencing libraries by comparing all quality trimmed reads against the cnidarian reference

148 protein collection from Kayal et al. (2018) using translated BLAST queries. After extraction of 
149 target reads and binning by locus, reads were assembled for each species using the SPAdes

150 assembler (version 3.10.1; Bankevich et al., 2012), followed by alignment and scaffolding of

151 contigs against the reference proteins using Exonerate (version 2.2.0; Slater \& Birney, 2005). In-

152 frame coding sequences of scaffolds (super-contigs) were translated into amino acids. All loci

153 were individually aligned using MAFFT (version 7.271; Katoh \& Standley, 2013) and

154 ambiguous alignment positions were removed using Gblocks (version 0.91b; Talavera \&

155 Castarena, 2007) via Gblockwrapper (version 0.03; https://goo.gl/fDjan6). Gene trees and their

156 support from 1,000 non-parametric bootstrap replicates were inferred using RAxML (version

157 8.2.12; Stamatakis, 2006) under the best fit model of sequence evolution (LG, WAG, or MtZoa;

158 see phylogenetic inference below) using the computational resources provided by the Open

159 Science Grid (Pordes et al., 2007; Sfiligoi et al., 2009). Gene trees were summarized by

160 maximizing the number of shared quartet trees using ASTRAL-III (version 5.7.7; Zhang et al.,

161 2018). Using the resulting phylogenetic tree and quartet frequenceies enabled us to identify areas

162 of gene tree discordance. Following alignment and removal of ambiguous alignment positions,

163 all loci were concatenated for further phylogenetic analyses.

164

165

166

167

168

169

170

171

172

\section{Phylogenetic inference}

Phylobayes (version 4.1c; Lartillot et al., 2013) was used to run eight independent MCMC chains and the posterior probability distribution was sampled until chains converged and a large sample of trees was generated. To account for site-specific differences in the evolutionary rates within and among alignment partitions, site-specific rates were inferred from the data using the CATGTR model during MCMC runs (Lartillot \& Philippe, 2004). PartitionFinder (version 2.1.1; Lanfear et al., 2017) was used to determine the best partitioning scheme for the concatenated alignment, with maximum likelihood trees for the partitioning analysis inferred using RAxML 
173 (version 8.2.12; Stamatakis, 2006). In the absence of prior information on possible partitioning

174 schemes, the relaxed clustering algorithm (rclusterf; Lanfear et al., 2014) was used to identify

175 partitioning schemes that fit the data well. Initial clustering analyses included all substitution

176 models implemented in PartitionFinder. These exploratory analyses failed to finish after more

177 than a month of run-time but indicated that models with rate heterogeneity modeled by drawing

178 from the gamma distribution $(+\mathrm{G})$ and amino acid residue equilibrium frequencies estimated

179 from the data $(+\mathrm{F})$ fit data partitions best. Due to their prevalence in preliminary results and

180 tractability of partitioning analysis, the final partitioning scheme was inferred using the LG (Le

181 \& Gascuel, 2008), WAG (Wheelan \& Goldman, 2001), and MtZoa (Rota-Stabelli et al., 2009)

182 substitution matrices. The resulting partitioning scheme was used to infer the maximum

183 likelihood phylogeny using RAxML (Stamatakis, 2006). The best tree was chosen from a set of

18410 trees inferred from independent searches, starting from different random starting trees.

185 Robustness of the resulting maximum likelihood phylogeny was assessed using 681 non-

186 parametric bootstrap replicates.

\section{Tree topology hypothesis testing}

189 Minimally constrained maximum likelihood phylogenetic tree searches were conducted to

190 evaluate which lineage of Hydroidolina is the sister group to the remainder of Hydroidolina:

191 Aplanulata, Capitata (Cartwright \& Nawrocki 2015), or Siphonophorae (Kayal et al. 2015). In

192 addition, tree topologies were fully constrained following the phylogenetic hypotheses proposed

193 in this contribution and previous phylogenetic hypotheses of Hydroidolina (Cartwright \&

194 Nawrocki 2010; Kayal et al. 2015). To discriminate between competing hypotheses of

195 hydroidolinan relationships inferred under these comprehensive constraints, phylogenetic 
196 analyses were conducted using maximum likelihood followed by topological hypothesis tests. In

197 short, backbone nodes of each tree, including the best trees found in unconstrained searches

198 presented in this contribution, were fixed at nodes that define higher-level relationships of in-

199 and outgroups. Under these constraints, tree inferences were able to rearrange the topology of

200 unconstrained nodes and make adjustments to branch lengths to maximize the likelihood of the

201 tree given the data in the concatenated amino acid residue alignment. These $\backslash$ constrained

202 maximum likelihood phylogenies were reconstructed using 10 independent partitioned RAxML

203 searches, retaining the tree with the highest $\log$ likelihood $(\operatorname{lnL})$. Further, the two competing

204 hypotheses presented in this contribution were compared using fully constrained Bayesian

205 inferences. By using fully constrained starting phylogenies, we set informative priors on tree

206 searches sensu Bergstein et al. (2013). Samples of the Bayesian posterior probability distribution

207 were obtained from four independent MCMC chains under both the CAT-GTR and default CAT-

208 Poisson (F81) models implemented in PhyloBayes, as described under phylogenetic inference

209 above. Sampling the posterior of tree searches under two different models allowed us to identify

210 the sensitivity of the phylogenetic inference to model misspecifications.

211 The Bayesian inference presented herein was used as the null hypothesis $\left(\mathrm{T}_{0}\right)$ against which

212 all alternate trees were evaluated quantitatively using the likelihood ratio. The likelihood ratio

213 statistic was calculated as

$\delta \ln L=2\left(\ln L \mathrm{~T}_{\mathrm{A}}-\ln \mathrm{L} \mathrm{T}_{0}\right)$

215 where $\operatorname{lnL} \mathrm{T}_{0}$ represents the likelihood of the tree under the null hypothesis and $\operatorname{lnL} \mathrm{T}_{\mathrm{A}}$ the

216 likelihood of the tree under the alternate hypothesis. Here, a positive $\delta \ln L$ indicates a better fit of

$217 \mathrm{~T}_{0}$ to the data while a negative $\delta \ln L$ indicates that $\mathrm{T}_{\mathrm{A}}$ explains the data better. Resampling of site

$218 \log$ likelihoods (RELL; Kishino et al., 1990; Hasegawa \& Kishino, 1994) was used to generate 
21910,000 bootstrap samples for each tree hypothesis, estimating the variance of tree likelihoods.

220 Using these RELL bootstrap distributions, the null hypothesis that trees of the candidate set have

221 the same $\operatorname{lnL}$ was tested via the approximately unbiased test (AU; Shimodaira, 2002), as

222 implemented in Consel (version 0.2; Shimodaira \& Hasegawa, 2001). Phylogenetic tree fit to the

223 data was further evaluated using the posterior probability of all candidate trees using the

224 Bayesian Information Criterion (BIC; Schwarz, 1978) approximation implemented in Consel.

225 Bayes factors (reviewed in Morey et al., 2016) were calculated to compare the Bayesian

226 majority rule consesus topology $\left(\mathrm{T}_{0}\right)$ with the alternate maximum likelihood topology $\left(\mathrm{T}_{1}\right)$

227 inferred in this constribution under the CAT-GTR and CAT-Poisson (F81) models in pairwise

228 comparisons. These comparisons address the question of how well the best tree hypothesis

229 predicts the observed amino acid alignment compared to the alternate tree hypotheses under a

230 given substitution model. Bayesian model evaluation requires quantifying model evidence by a

231 marginal likelihood function through integration of the product of the likelihood and the prior

232 (Fourment et al., 2020). Model comparisons with Bayes factors use ratios of marginalized

233 likelihoods, which are difficult to compute exactly. To address this issue, the harmonic mean of

234 the posterior is widely used as an estimator of the marginal likelihood (Kass \& Raftery, 1995).

235 Combining the posterior distributions of four MCMC chains for a given constrained tree search,

236 we calculated the moving harmonic mean of the posterior using a sliding window with a step

237 length of one and a size equal to the current MCMC cycle times 0.33 . The natural logarithm of

238 the ratio of harmonic means was used for tree comparisons and interpreted following a modified

239 version of Jeffreys' (1961) categories of evidence (cf. Kass and Raftery, 1995). In particular,

240 Bayes factors were calculated as

$\mathrm{BF}_{10}=2 * \ln \left(\operatorname{lnL} \mathrm{T}_{1}-\ln L \mathrm{~T}_{0}\right)$ 
242 where $\operatorname{lnL} \mathrm{T}_{0}$ is the marginal likelihood of the MCMC search under the null hypothesis and $\mathrm{T}_{1}$

243 the marginal likelihood of the alternate MCMC tree search. Note that the likelihood ratio may be

244 negative leading to an undefined result of the logarithmic function, an issue we took into account

245 by taking the absolute of the ratio if negative, followed by multiplying the resulting Bayes factor

246 by negative one.

247

248 Results

249 Recovery of target loci and alignment

250 The final concatenated alignment (Fig. 1A; Supplementary File 2) contained 44 medusozoan

251 cnidarians, sequence data for 18 of which were generated in this study (Table 2). This alignment

252 contained 134 of the 355 targeted loci, with a total alignment length of 21,816 character

253 columns. Loci targeted but not included in the final alignment failed to generate sequence data

254 from target enrichment reactions and were excluded from further analysis. Alignment lengths per

255 locus ranged from 56 to 363 positions (average 164), with a combined total of 333 to 5,818

256 (average 2,891) amino acids contained in each alignment (Fig. 1B). Taxon occupancy per locus

257 ranged from 5 to 36 species with an average of 21 species included in each alignment partition

258 for phylogenetic analysis (Fig. 1C). Among ingroup hydrozoans, coverage varied between and

259 within clades, with matrix completeness being highest for taxa of the Kayal et al. (2018)

260 reference dataset (Fig. 1A). In particular, sequence data for Aplanulata, Siphonophorae, and

261 Filifera III largely represent previously published sequence data that were used for bait design

262 (Table 2). Consequently, alignment coverage in these groups ranges from some $40 \%$ to almost

$26380 \%$. Despite being closely related to at least some of the taxa used in bait design, success in

264 recovering loci from Podocoryne martinicana (Filifera III) and Athorybia rosacea 
265 (Siphonophorae) was limited (Table 2). In species of Filifera I, Filifera II, and Capitata target

266 capture yields ranged from as few as some 500 amino acid residues spread over five loci

267 (Myrionema hargitti) to as high as some 4,400 residues contained in 46 separate loci (Pennaria

268 disticha). By contrast, the RNA bait set and hybridzation protocol employed here was highly

269 successful in recovering sequence data for leptothecate hydrozoans despite being somewhat

270 distantly related to any of the reference taxa. On average, 49 target loci comprising close to

2715,000 amino acid residues were recovered for leptothecate species (Table 2). Here, the maximum

272 yield was some 8,000 residues spread across 80 separate loci (Kirchenpaueria sp.). Consistent

273 with the high degree of missing sequence data, gene trees displayed a large amount of

274 discordance for both ingroup and outgroup taxa (Fig. 2).

275

276

Phylogeny of Hydroidolina inferred from target-capture sequencing

277 Bayesian MCMC chains using the CAT-GTR model were terminated after some 14,000

278 iterations, of which the first 3,000 were discarded as burn-in. Chains were thinned by sampling

279 every tenth generation, yielding a mean difference between chains of 9e-4 and a maximum

280 difference of 0.0469 . The majority rule consensus of the posterior shows a well-resolved

281 phylogeny with high posterior probabilities for the monophyly of the major ingroup and

282 outgroup clades (Fig. 3). Aplanulata represents the sister group to the remainder of Hydroidolina.

283 As in previous analyses, Filifera represents a polyphyletic taxon. Interestingly, Filifera I plus II

284 are each other's closest relative as are Filifera III plus IV. Filifera I plus II are the closest

285 relatives to Capitata, albeit with a posterior probability slightly less than 0.95 ; Leptothecata is

286 inferred as sister to the clade of Filifera I plus II and Capiata. Filifera III plus IV are the closest

287 relatives of Siphonophorae. 
For ML phylogenetic inference, the best partitioning scheme of the 134 locus dataset

289

290

291

292

293

294

295

296

297

298

299

300

301

302

303

304

305

306

307

308

309

310

contained 25 partitions. In particular, the ML phylogeny was inferred using 22 partitions

comprising 128 loci under the $\mathrm{LG}+\mathrm{G}+\mathrm{F}$ model, two partitions with a total of 5 loci under the

$\mathrm{MTZOA}+\mathrm{G}+\mathrm{F}$ model, and 1 partition with a single locus under the $\mathrm{WAG}+\mathrm{G}+\mathrm{F}$ model. The $\mathrm{ML}$

tree largely agrees with the Bayesian inference (Fig. 3), but differs in the placement of Filifera I

plus II relative to Capitata and Leptothecata. In contrast to the Bayesian inference, Filifera I plus

II are the sister to Leptothecata rather than Capitata. However, this result lacks support with a

bootstrap of less than 50 . While the majority of the other nodes in both the in- and outgroup

received high bootstrap support, the deep ingroup node uniting Filifera III plus IV and

Siphonophorae is weakly supported with a bootstrap of 65. Despite these differences between

Bayesian and ML phylogenies, both analyses agree in confidently inferring Aplanulata to be the sister lineage of the rest of Hydroidolina.

\section{Phylogenetic hypothesis testing}

Aplanulata represents the most likely sister taxon to the remainder of Hydroidolina (Fig. 4 left)

with the alternate hypotheses of Capitata (Fig. 4 center) or Siphonophorae (Fig. 4 right) both

receiving low BIC posterior probabilities and being rejected by the AU test. Fully constrained

phylogenetic inferences provided further insight into the relationships within Hydroidolina (Fig.

5A). Two phylogenies, $T_{2}$ (cf. Cartwright \& Nawrocki, 2010) and $\mathrm{T}_{3}$ (cf. Kayal et al., 2015),

were rejected by the AU test (Table 3). Interestingly, $\mathrm{T}_{2}$ had a higher likelihood than $\mathrm{T}_{3}$, indicating a worse fit of $\mathrm{T}_{3}$ to the data compared to $\mathrm{T}_{2}$. $\mathrm{T}_{0}$ and $\mathrm{T}_{1}$ were constrained following the

Bayesian and Maximum likelihood phylogenies inferred herein (Fig. 3). Both tree hypotheses are roughly equally likely, with the maximum likelihood phylogeny $\mathrm{T}_{1}$ being a slightly better fit to

Peer) reviewing PDF | (2020:11:55841:2:0:NEW 1 Aug 2021) 
311 the data using maximum likelihood tree searches (Table 3). Constrained Bayesian searches using

$312 \mathrm{~T}_{0}$ and $\mathrm{T}_{1}$ as priors generated a sample of 27,000 $\log$ likelihoods from the posterior, with chains

313 mixing well after burn-in (Fig. 5B). The marginal likelihood of $\mathrm{T}_{0}$ was higher than the marginal

314 likelihood of $\mathrm{T}_{1}$ under the GTR model while the marginal likelihood of $\mathrm{T}_{1}$ was higher under the

315 F81 model (Fig. 5C). Under the GTR substitution model, $\mathrm{T}_{0}$ is better at predicting the data than

$316 \mathrm{~T}_{1}\left(\mathrm{BF}_{10}=10.48\right.$; Fig. $\left.5 \mathrm{D}\right)$. By contrast, $\mathrm{T}_{1}$ is favored under the $\mathrm{F} 81$ model $\left(\mathrm{BF}_{10}=-8.84\right.$; Fig.

317 5D).

318

319 Discussion

320 A new phylogenetic hypothesis for Hydroidolina

321 Despite highly variable recovery rates of target loci that leave large gaps in the final alignment

322 (Fig. 1A), the dataset analyzed here provides high resolution of the deep phylogeny of

323 Hydroidolina (Fig. 3) even though discordance among gene trees is apparent (Fig. 2).

324 Considering the ancient radiation of medusozoan cnidarians, the gene tree discordance observed

325 here is unlikely a result of incomplete lineage sorting but rather the result of gene tree estimation

326 error driven by the limited information contained in individual amino acid alignments.

327 Coalescent-based summary methods lack accuracy in the presence of substantial gene tree

328 estimation error (Warnow, 2015) and, under these conditions, phylogenetic analyses of fully

329 partitioned concatenated alignments are preferable over summary methods that implement multi-

330 species coalescent models (cf. Molloy \& Warnow, 2018; Bryant \& Hahn, 2020). While missing

331 data could affect tree topology inference from concatenated alignments, in practice accurately

332 placing taxa despite missing information is often not a major concern (Wiens et al., 2011).

333 Indeed, we were able to infer a well-resolved phylogeny by combining a publicly available data- 
334 rich amino acid sequence dataset with new data from key hydrozoan taxa that have so far been

335 absent from multi-gene phylogenomic datasets. Overall topologies recovered in our phylogenetic

336 inferences are consistent with previous phylogenomic analyses (Zapata et al., 2015; Kayal et al.,

337 2018). While neither Zapata et al. (2015) nor Kayal et al. (2018) included sufficient taxa

338 representing Hydroidolina to allow for much insight into the evolutionary history of the group,

339 both recovered Aplanulata as the sister clade of the remaining hydroidolinan groups, a placement

340 we confirmed after comprehensively sampling higher-level taxa of Hydroidolina (Fig. 3). This

341 placement is at odds with previous phylogenetic treatments of Hydroidolina that inferred a more

342 recent origin of Aplanulata within Hydroidolina as sister of Filifera I plus II (Cartwright \&

343 Nawrocki, 2010; Kayal et al., 2015). Placing Aplanulata as the sister-group of Hydroidolina has

344 potentially far-reaching consequences for our understanding of hydrozoan evolution. Aplanulata

345 is dominated by solitary polyp-forming taxa, a trait common in other medusozoans but rare

346 across Hydroidolina in which colonial hydroids, many of which are polymorphic, displaying a

347 reproductive division of labor, dominate (Cartwright \& Nawrocki, 2010; Cartwright et al., 2020).

348 As such, the placement of Aplanulata as sister to the remainder of Hydroidolina suggests that the

349 last common ancestor of Hydroidolina possessed a solitary polyp that produces medusae as part

350 of a metagenetic life cycle in which an asexually reproducing polyp generation alternates with a

351 sexually reproducing medusa generation (cf. Bentlage et al., 2018). Pending formal analyses

352 using ancestral state reconstructions with greater taxon sampling, the evolution of coloniality and

353 division of labor, including losses of metagenesis (lack of free-swimming medusae), are likely

354 derived traits in Hydroidolina.

355 Ingroup relationships within Hydroidolina appear largely congruent across the Bayesian

356 posterior tree set and the maximum likelihood phylogeny with the exception of the placement of 
357 Filifera I plus II with respect to Capitata and Leptothecata (Fig. 3). One possible explanation for

358 this disagreement may be that the substitution matrix-based models used for maximum

359 likelihood inference here are potentially less accurate in reflecting the substitution process that

360 led to the observed data. Exploratory partitioning analyses with PartitionFinder suggested that

361 substitutions in a significant number of partitions should be inferred using the GTR model.

362 However, benchmarking of partitioning analyses under the GTR model indicated that

363 computational time would be prohibitive to search across this large parameter space. To address

364 this issue, partitioning schemes were inferred using simpler substitution matrices (i.e., LG,

365 WAG, and MtZoa). Regardless of this poorly supported topological difference in the placement

366 of Filifera I plus II (Fig. 3), the overall relationships recovered are consistent across analyses but

367 at odds with previous phylogenetic hypotheses of Hydroidolina (cf. Cartwright \& Nawrocki,

368 2010; Kayal et al., 2015). Despite conflicts between the phylogenetic hypotheses presented here

369 and previous treatments of Hydroidolina, a consistent result that has emerged over the last

370 decade is the polyphyly of Filifera. In agreement with prior phylogenetic hypotheses (Cartwright

371 \& Nawrocki, 2010; Kayal et al., 2015), we find that Filifera I plus II forms a monophyletic clade,

372 as does Filifera III plus IV. As of now, these clades remain without clear definition based on

373 morphological or life history characteristics but Cartwight et al. (2008) provide the most detailed

374 discussion of possible synapomorphies of hydroidolinan clades to date.

375

376 Phylogenetic tree selection

377 Our results appear unequivocal on the position of Aplanulata as sister to the remaining lineages

378 of Hydroidolina (Figs 2-4). To facilitate discriminating between competing hypotheses of

379 hydroidolinan relationships, we quantified the evidence for the set of alternate phylogenetic 
380 hypotheses (Fig. 5A) in light of the multi-locus dataset analyzed here. Likelihood ratio statistics

381 (Table 3) indicate that the phylogenies proposed in this contribution (Fig. 3; Fig. 5A $\mathrm{T}_{0}$ and $\mathrm{T}_{1}$ )

382 are a better fit than either the previously published rDNA (Fig. 5A $\mathrm{T}_{2}$; Cartwright \& Nawrocki,

383 2010) or mitochondrial genome-based (Fig. 5A T3; Kayal et al., 2015) phylogenetic hypotheses.

384 Indeed, $\mathrm{T}_{2}$ and $\mathrm{T}_{3}$ were rejected by the $\mathrm{AU}$ test while neither $\mathrm{T}_{0}$ nor $\mathrm{T}_{1}$ can be rejected (Table 3 ).

385 In addition, the majority rule consensus of the Bayesian posterior $\left(\mathrm{T}_{0}\right)$ and the maximum

386 likelihood topology $\left(\mathrm{T}_{1}\right)$ received high BIC-based posterior probabilities compared to the very

387 small posterior probabilities of the alternate hypotheses (Table 3).

388 The hypothesis tests above reduced the set of credible trees by eliminating unlikely tree

389 topologies. The procedures employed rely on the likelihood of a single phylogeny, implicitly

390 assuming that the tree is known without error. Resampling of site likelihoods from this best tree

391 was used to estimate variances of tree likelihoods for comparisons. Bayesian approaches that

392 estimate the posterior probability distribution of phylogenetic hypotheses allow averaging across

393 tree topologies and branch lengths for more comprehensive incorporation of uncertainty into

394 comparisons of phylogenetic trees but may be affected adversely by misspecifications of the

395 prior (e.g., Bergstein et al., 2013). Bayes factors incorporate phylogenetic uncertainty in model

396 comparisons by employing ratios of marginal likelihoods and allowed us to quantify the weight

397 of evidence for a topological hypothesis under a given amino acid substitution model. We found

398 that $\mathrm{T}_{0}$ predicts the alignment data best under the GTR model while $\mathrm{T}_{1}$ predicts the alignment

399 best under the simpler F81 model (Fig. 5C \& D). The difference between these two topologies is

400 limited to the placement of Filifera I plus II with respect to Capitata and Leptothecata (Fig. 3)

401 and we suspect that the sensitivity to model choice may be driven by the lack of data for Filifera

402 I plus II (Fig. 1) and the resulting gene tree discordance (Fig. 2). 
Despite being unable to confidently choose a single phylogenetic hypothesis as fitting the

404 data best using maximum likelihood and Bayesian hypothesis testing frameworks, the

405 phylogenetic hypotheses presented in this contribution (Fig. 3) consistently display high

406 predictive power for the alignment data compared to the alternate hypotheses proposed

407 previously. Consequently, we suggest that our phylogenetic framework for Hydroidolina

408 represents the most viable hypothesis of hydroidolinan relationships to date. That said, further

409 studies with additional taxa and characters are still needed to assess the validity of this working

410 hypothesis and clarify the affinities of Filifera I plus II in particular. Such analyses will also

411 allow for more thorough analyses of the evolution of life history characteristics across this most

412 speciose of medusozoan clades.

413

\section{Conclusions}

415 This study represents one of only a few attempts at using target-enriched high throughput

416 sequencing to generate a multi-locus alignment for phylogenetic analyses of Cnidaria. Previous

417 attempts had been limited to Anthozoa (Quattrini et al., 2018; Erickson et al., 2020), relying on

418 the greater availability of genomic data for Anthozoa compared to Medusozoa. Despite mixed

419 success in capturing target loci, we were able to generate an informative multi-gene alignment

420 that produced a well-supported phylogenetic hypothesis for Hydroidolina. One drawback of bait-

421 development based on coding sequences alone, as done here, is that baits may inadvertently be

422 designed across intron-exon boundaries which is likely to reduce bait effectiveness. As

423 availability of genomic resources increases, it will be possible to address these issues and apply

424 target capture sequencing approaches routinely to phylogenetic studies of medusozoan

425 cnidarians, building on the growing knowledge-base for designing targeted high-throughput 
426 sequencing experiments (reviewed in Andermann et al., 2020). Nonetheless, we were able to

427 address long-standing questions in hydrozoan phylogenetics by applying relevant constraints for

428 discriminating between alternate phylogenetic hypotheses for Hydroidolina. We suggest that the

429 consensus of $\mathrm{T}_{0}$ and $\mathrm{T}_{1}$ (Fig. 5A) represents the most likely tree topology for hydroidolinan

430 evolutionary relationships to date. While the likelihood-based AU test (Table 3) allowed

431 rejection of several alternate topologies, Bayes factors (Fig. 5D) suggested that the choice of

432 substitution model affects the posterior distributions of the two alternate topologies inferred. As

433 such, the most viable representation of phylogenetic relationships of Hydroidolina is the

434 consensus of the two topologies inferred herein.

435

436 Acknowledgements

437 This work was supported by Guam EPSCoR through NSF awards OIA-1457769 and OIA-

438 1946352, and funding by the Small Grants program of the National Museum of Natural History

439 (NMNH), Smithsonian Institution. Part of this work was performed using resources of the

440 Laboratories of Analytical Biology (LAB) at NMNH and we thank LAB staff for valuable advice

441 on laboratory protocols and procedures. The Open Science Grid (NSF award 2030508) and its

442 support staff of research computing facilitators enabled high throughput computing for

443 phylogenetic inferences. BB wishes to acknowledge the numerous discussions with the

444 participants in his bioinformatics and data analysis course that informed many of the analytical

445 approaches for phylogenetic hypothesis testing used here. We thank Casey Dunn and an

446 anonymous reviewer for suggestions that improved this work. 


\section{References}

448 Andermann T, Torres JMF, Matos-Maraví P, Batista R, Blanco-Pastor JL, Gustafsson ALS, 449 Kistler L, Liberal IM, Oxelman B, Bacon CD, Antonelli A (2020) A guide to carrying out a $450 \quad$ phylogenomic target sequence capture project. Frontiers in Genetics 10:1407.

451 Bankevich, A., Nurk, S., Antipov, D., Gurevich, A. A., Dvorkin, M., Kulikov, A. S., Lesin, V. 452 M., Nikolenko, S. I., Pham, S., Prjibelski, A. D., Pyshkin, A. V., Sirotkin, A. V., Vyahhi, N., 453 Tesler, G., Alekseyev, M. A., Pevzner, P. A. (2012) SPAdes: A New Genome Assembly 454 Algorithm and Its Applications to Single-Cell Sequencing. Journal of Computational $455 \quad$ Biology, 9: 455-477.

456 Bayha, K. M., Collins, A. G., \& Gaffney, P. M. (2017). Multigene phylogeny of the scyphozoan 457 jellyfish family Pelagiidae reveals that the common U.S. Atlantic sea nettle comprises two distinct species (Chrysaora quinquecirrha and C. chesapeakei). PeerJ, 5, e3863.

Bentlage B, Osborn KJ, Lindsay DJ, Hopcroft RR, Raskoff KA, Collins AG (2018) Loss of metagenesis and evolution of a parasitic life style in a group of open-ocean jellyfish. Mol Phyl Evol 124:50-59.

Bergsten J, Nilsson AN, Ronquist F (2013) Bayesian tests of topology hypotheses with an example from diving beetles. Systematic Biology 62:660-673.

Bolger, A. M., Lohse, M., \& Usadel, B. (2014). Trimmomatic: A flexible trimmer for Illumina Sequence Data. Bioinformatics 30:2114-2120.

Brekhman V, Malik A, Haas B, Sher N, Lotan T (2015) Transcriptome profiling of the dynamic life cycle of the scypohozoan jellyfish Aurelia aurita. BMC Genomics 14:74. 
468 Bryant D, Hahn MW (2020) The Concatenation Question. Phylogenetics in the Genomic Era

469 (Eds: Scornavacca C, Delsuc F, Galtier N), No commercial publisher, Authors open access

$470 \quad$ book, pp. 3.4:1-3.4:23. (hal-02535651)

471 Cartwright P, Evans NM, Dunn CW, Marques AC, Miglietta MP, Collins AG. 2008.

472 Phylogenetics of

473 Hydroidolina (Cnidaria, Hydrozoa). J Mar Biol Assoc 88:1163-72.

474 Cartwright P, Halgedahl SL, Hendricks JR, Jarrard RD, Marques AC, Collins AG, Lieberman

475 BS (2007) Exceptionally preserved jellyfishes from the middle Cambrian. PloS One 2:e1121.

476 Cartwright, P., Nawrocki, A.M., 2010. Character evolution in Hydrozoa (phylum Cnidaria).

$477 \quad$ Integr Comp Biol 50, 456-472.

478 Cartwright P, Travert MK, Sanders SM (2020) The evolution of development of coloniality of

479 hydrozoans. J Exp Zool DOI: 10.1002/jez.b.22996

480 Collins AG, Schuchert P, Marwues, AC, Jankowski T, Medina M, Schierwater B (2006)

481 Medusozoan phylogeny and character evolution clarified by new large and small subunit

482 rDNA data and assessment of the utility of phylogenetic mixture models. Systematic Biology, $483 \quad 55,97-115$.

484 Chapman JA, Kirkness EF, Simakov O, Hampson SE, Mitros T, Weinmaier T, Rattei T,

485 Balasubramanian PG, Borman J, Busam D, Disbennett K, Pfannkoch C, Sumin N, Sutton

486 GG, Viswanathan LD, Walenz B, Goodstein DM, Hellsten U, Kawashima T, Prochnik SE,

487 Putnam NH, Shu S, Blumberg B, Dana CE, Gee L, Kibler DF, Law L, Lindgens D, Martinez

488 DE, Peng J, Wigge PA, Bertulat B, Guder C, Nakamura Y, Ozbek S, Watanabe H, Khalturin

489 K, Hemmrich G, Franke A, Augustin R, Fraune S, Hayakawa E, Hayakawa S, Hirose M,

490 Hwang JS, Ikeo K, Nishimiya-Fujisawa C, Ogura A, Takahashi T, Steinmetz PRH, Zhang X, 
491

492

493

494

495

496

497

498

499

500

501

502

503

504

505

506

507

508

509

510

511 before and during the Cambrian explosion. In: The Cnidaria, Past, Present, and Future (eds. 512 Goffredo S, Dubinsky Z), 15-29.

Aufschnaiter R, Eder M-K, Gorny A-K, Salvenmoser W, Heimberg AM, Wheeler BM, Peterson KJ, Böttger A, Tischler P, Wolf A, Gojobori T, Remington KA, Strausberg RL, Venter JC, Technau U, Hobmayer B, Bosch TCG, Holstein TW, Fujisawa T, Bode HR, David CN, Rokhsar DS, Steele RE (2010) The dynamic genome of Hydra. Nature 464:592596.

Daly M, Brugler MR, Cartwright P, Collins AG, Dawson MN, Fautin DG, France SC, McFadden CS, Opresko DM, Rodriguez E, Romano SL, Stake JL (2007) The phylum Cnidaria: a review of phylogenetic patterns and diversity 300 years after Linnaeus. Zootaxa 1668:127-182.

Erickson KL, Pentico1 A, Quattrini AM, McFadden CS (2020) New approaches to species delimitation and population structure of corals: two case studies using ultra-conserved elements and exons. Molecular Ecology Resources DOI: 10.1111/1755-0998.13241.

Erwin DH (2020) The origin of animal body plans: a view from fossil evidence and the regulatory genome. Development 147:dev182899.

Fourment M, Magee AF, Whidden C, Bilge A, Matsen FA, Minin VN (2020) 19 dubious ways to compute the marginal likelihood of a phylogenetic tree topology. Systematic Biology 69:209-220.

Green MR, Sambrook J (2012) Molecular Cloning - A laboratory manual. Cold Spring Harbor Press, Cold Spring Harbor, NY, 2028pp.

Han J, Zang X, Komiya T (2016) Integrated evolution of cnidarians and oceanic geochemistry 
513 Hasegawa M, Kishino H. (1994) Accuracies of the simple methods for estimating the bootstrap

514 probability of a maximum-likelihood tree. Mol Biol Evol 11:142-145.

515 Jeffreys, H. (1961). Theory of probability (3rd ed.). Oxford University Press, New York, NY, $516 \quad 472 \mathrm{pp}$.

517 Johnson MG, Gardner EM, Liu Y, Medina R, Goffinet B, Shaw AJ, Zerega NJC, Wickett NJ

518 (2016) HybPiper: Extracting coding sequence and introns for phylogenetics from high-

519 throughput sequencing reads using target enrichment. Applications in Plant Sciences, 4.

520 Kass RE, Raftery AE (1995) Bayes factors. Journal of the American Statistical Association

$521 \quad 430: 773-795$.

522 Katoh, K., \& Standley, D. M. (2013). MAFFT multiple sequence alignment software version 7:

523 improvements in performance and usability. Mol Biol Evol 30:772-780.

524 Kayal E, Bentlage B, Cartwright P, Yanagihara AA, Lindsay DJ, Hopcroft RR, Collins AG

525 (2015) Phylogenetic analysis of higher-level relationships within Hydroidolina (Cnidaria:

526 Hydrozoa) using mitochondrial genome data and insight into their mitochondrial

527 transcription. PeerJ 3:e1403.

528 Kayal E, Bentlage B, Pankey MS, Ohdera AH, Medina M, Plachetzki DC, Collins AG, Ryan JF

529 (2018) Phylogenomics provides a robust topology of the major cnidarian lineages and

$530 \quad$ insights on the origins of key organismal traits. BMC Evolutionary Biology 18:68.

531 Kishino H, Miyata T, Hasegawa M (1990) Maximum-likelihood inference of protein phylogeny

532 and the origin of chloroplasts. J Mol Evol 31:151-160.

533 Lanfear, R., Calcott, B., Kainer, D., Mayer, C., \& Stamatakis, A. (2014). Selecting optimal

534 partitioning schemes for phylogenomic datasets. BMC Evolutionary Biology 14: 82. 
535 Lanfear, R., Frandsen, P. B., Wright, A. M., Senfeld, T., Calcott, B. (2017) PartitionFinder 2:

536 new methods for selecting partitioned models of evolution for molecular and morphological

537 phylogenetic analyses. Mol Biol Evol 34:772-773.

538 Lartillot N, Philippe H (2004) A Bayesian mixture model for across-site heterogeneities in the 539 amino-acid replacement process. Mol Biol Evol 21:1095-1109.

540 Lartillot N, Rodrigue N, Stubbs D, Richer J (2013) PhyloBayes MPI: Phylogenetic

541 reconstruction with infinite mixtures of profiles in a parallel environment. Systematic Biology

$542 \quad 62: 611-615$.

543 Le, SQ, Gascuel, O. 2008 An improved general amino-acid replacement matrix. Mol Biol Evol $544 \quad 25: 1307-1320$.

545 Mirabab S (2019) Species tree estimation using ASTRAL: Practical Considerations. arXiv $546 \quad 1904.03826 v 2$.

547 Molloy EK, Warnow T (2018) To include or not to include: The impact of gene filtering on $548 \quad$ species tree estimation methods. Systematics Biology 67:285-303.

549 Morey RD, Romeijn J-W, Rouder JN (2016) The philosophy of Bayes factors and the 550 quantification of statistical evidence. Journal of Mathematical Psychology 72:6-18.

551 Ohdera A, Ames CL, Dikow RB, Kayal E, Chiodin M, Busby B, La S, Pirro S, Collins AG, 552 Medina M, Ryan JF. (2019) Box, stalked, and upside-down? Draft genomes from diverse 553 jellyfish (Cnidaria, Acraspeda) lineages: Alatina alata (Cubozoa), Calvadosia cruxmelitensis

554 (Staurozoa), and Cassiopea xamachana (Scyphozoa). GigaScience 8:giz069.

555 Pordes R, Petravick D, Kramer B, Olson D, Livny M, Roy A, Avery P, Blackburn K, Wenaus

556 T, Würthwein F, Foster I, Gardner R, Wilde M, Blatecky A, McGee J, Quick R (2007) The 557 open science grid. Journal of Physics: Conference Series 78:012057. 
558 Putnam NH, Srivastava M, Hellsten U, Dirks B, Chapman J, Salamov A, Terry A, Shapiro A,

559 Lindquist E, Kapitonov VV, Jurka J, Genikhovich G, Grigoriev IV, Lucas SM, Steele RE,

560 Finnerty JR, Technau U, Martindale MQ, Rokhsar DS (2007) Sea anemone genome reveals

561 ancestral eumetazoan gene repertoire and genomic organization. Science 317:86-94.

562 Quattrini AM, Faircloth BC, Dueñas LF, Bridge TCL, Brugler MR, Calixto-Botía IF, DeLeo

563 DM, Forêt S, Herrera S, Lee SMY, Miller DJ, Prada C, Rádis-Baptista G, Ramírez-Portilla

564 C, Sánchez JA, Rodríguez E, McFadden CS (2018) Universal target-enrichment baits for

565 anthozoan (Cnidaria) phylogenomics: New approaches to long-standing problems. Mol Ecol

566 Resour 18:281-295.

567 Rota-Stabelli O, Yang Z, Telford MJ. (2009) MtZoa: a general mitochondrial amino acid

568 substitutions model for animal evolutionary studies. Mol Phylogenet Evol. 52:268-272.

569 Rubinoff D, Holland BS (2005) Between two extremes: mitochondrial DNA is neither the

570 Panacea nor the nemesis of phylogenetic and taxonomic inference. Systematic Biology

$571 \quad 54: 952-961$.

572 Schuchert, P. (2020). World Hydrozoa Database. Accessed at

573 http://www.marinespecies.org/hydrozoa on 2020-09-15. DOI:10.14284/357

574 Schwarz G (1978) Estimating the dimension of a model. The Annals of Statistics 6:461-464.

575 Sfiligoi I, Bradley DC, Holzman B, Mhashilkar P, Padhi S, Würthwein F (2009) The pilot way to

576 grid resources using glideinWMS. 2009 WRI World Congress on Computer Science and

577 Information Engineering 2:428-432.

578 Shimodaira, H. (2002) An approximately unbiased test of phylogenetic treeselection. Systematic

579 Biology 51:492-508. 
580 Shimodaira, H. and Hasegawa, M. (2001) CONSEL: for assessing the confidence of

581 phylogenetic tree selection. Bioinformatics 17:1246-1247.

582 Shinzato, C., Shoguchi, E., Kawashima, T., Hamada, M., Hisata, K., Tanaka, M., Fujie, M.,

583 Fujiwara, M., Koyanagi, R., Ikuta, T., Fujiyama, A., Miller, D. J., and Satoh, N. (2011)

584 Using the Acropora digitifera genome to understand coral responses to environmental

585 change. Nature 476:320-323.

586 Sober E (2008) Evidence and Evolution: The logic behind the science. Cambridge University

587 Press, Cambridge, UK, pp. 412.

588 Slater GS, Birney E (2005) Automated generation of heuristics for biological sequence

589 comparison. BMC Bioinformatics 6:31.

590 Smit AFA, Hubley R, Green P. (2013-2015) RepeatMasker Open-4.0

$591<$ http://www.repeatmasker.org $>$.

592 Stamatakis A (2006) RAxML-VI-HPC: maximum likelihood-based phylogenetic analyses with 593 thousands of taxa and mixed models, Bioinformatics 22:2688-2690.

594 Talavera, G., Castresana, J. (2007) Improvement of phylogenies after removing divergent and 595 ambiguously aligned blocks from protein sequence alignments. Systematic Biology 56: 564 596577.

597 Valentine JW, Jablonski D, Erwin DH (1999) Fossils, molecules and embryos: new perspectives 598 on the Cambrian explosion. Development 126:851-859.

599 Warnow T (2015) Concatenation analyses in the presence of incomplete lineage sorting. PLOS $600 \quad$ Currents: Tree of Life 1.

601 Wiens JJ, Morrill MC (2011) Missing data in phylogenetic analysis: reconciling results from 602 simulations and empirical data. Systematic Biology 60:719-731. 
603 Whelan, S. \& Goldman, N. 2001 A general empirical model of protein evolution derived from 604 multiple protein families using a maximum-likelihood approach. Mol Biol Evol18:691-699. 605 Zhang C, Rabiee M, Sayyari E, Mirarab S (2018) ASTRAL-III: Polynomial time species tree 606 reconstruction from partially resolved gene trees. BMC Bioinformatics 19: 153.

607 Zapata F, Goetz FE, Smith SA, Howison M, Siebert S, Church SH, Sanders SM, Ames CL, 608 McFadden CS, France SC, Daly M, Collins AG, Haddock SH, Dunn CW, Cartwright P 609 (2015) Phylogenomic analyses support traditional relationships within Cnidaria. PLoS One $610 \quad 10: \mathrm{e} 0139068$. 


\section{Figure Legends}

612 Fig. 1 A) Alignment of the 134 amino acid residue partitions; B) total number of amino acid

613 residues contained in each alignment partition; C) number of species included in each alignment

614 partition.

615

616 Fig. 2 Cladogram of statistical gene tree summary derived from the most common quartet trees

617 obtained from 134 bootstrapped gene trees. Node labels represent mean quartet frequencies and 618 give insight into areas of gene tree conflict in the dataset. Quartet frequencies of 1 indicate no

619 gene tree discordance while lower frequencies indicate discordance (Mirabab, 2019).

620

621 Fig. 3 Majority rule consensus of Bayesian phylogenetic inference under the CAT-GTR model 622 (left side) with posterior probabilities given at each node. Maximum likelihood phylogeny (right 623 side) was inferred from a partitioned analysis under the LG, WAG, and MtZoa models. Node

624 support values represent the summary of 681 non-parametric maximum likelihood bootstrap 625 searches. Scale bars indicate the number of substitutions per site. Scale bars indicate 626 substitutions per site on each respective phylogram.

628 Fig. 4 Minimally constrained maximum likelihood phylograms. Constrained nodes are indicated 629 by solid circles and the log likelihood for each phylogeny are given; scale bars indicates 630 substitutions per site. The backbone of the phylogeny was constrained to maintain monophyly of 631 the outgroup and monophyly of Hydroidolina with respect to Trachylina. Within Hydroidolina, 632 three hypotheses of sister-group relationships were evaluated: Aplanulata (left), Capitata 633 (center), or Siphonophorae (right) as sister to the remainder of Hydroidolina. The approximately 
634 unbiased test (AU) indicated that the Capitata (center) and Siphonophorae (right) sister-group

635 hypotheses should be rejected. The Bayesian Information Criterion (BIC) showed a good fit of

636 the Aplanulata (left) sister-group phylogeny to the alignment data.

637

638 Fig. 5 A) Phylograms inferred using fully constrained maximum likelihood searches to compare

639 explicit hypotheses of tree topologies; constraints indicated by solid black circles; scale bar

640 indicates substitutions per site. Tree topologies were constrained to reflect the Bayesian

641 inference $\left(T_{0}\right)$ and maximum likelihood phylogenies $\left(T_{1}\right)$ presented in this contribution (Fig. 3).

642 Two alternate tree searches were constrained to reflect previously published phylogenies $\left(\mathrm{T}_{2}\right.$ :

643 Cartwright \& Nawrocki, 2010; T3: Kayal et al., 2015); *** indicates a p-value of less than 0.001

644 for the approximately unbiased (AU) test (Table 3). Shallow nodes were collapsed and species

645 labels removed for legibility. Scale bar indicates the number of substitutions per site. B) Each

646 graph shows the log likelihoods of four independent Bayesian Markov Chain Monte Carlo

647 (MCMC) runs following burn-in. Log likelihoods for both $\mathrm{T}_{0}$ and $\mathrm{T}_{1}$ were sampled from the

648 posterior under the CAT-GTR and the CAT-Poisson (F81) model using the constraints shown in

649 (A). C) Harmonic means representing the marginal likelihood (white circles) and log likelihood

650 distributions of $\mathrm{T}_{0}$ and $\mathrm{T}_{1}$ under CAT-GTR (left) and CAT-Poisson (F81; right) models. Grey

651 lines indicate the harmonic means for $\mathrm{T}_{0}$. D) Bayes factors for pairwise comparisons between $\mathrm{T}_{0}$

652 and $\mathrm{T}_{1}(\mathrm{C})$. A negative Bayes factor provides evidence for $\mathrm{T}_{0}$ while a positive Bayes factor

653 indicates evidence against $\mathrm{T}_{0}$, providing support for $\mathrm{T}_{1}$. A Bayes factor between -2 and 2

654 indicates lack of evidence or no evidence favoring one hypothesis over the other, Bayes factors

655 in the range of 2 to 6 ( -2 to -6$)$ provide some evidence, 6 to 10 (-6 to -10$)$ strong evidence, and

656 Bayes factors $>10(<-10)$ very strong evidence for one hypothesis compared to the other. 
657 Table 1 Reference genomes used for verification of candidate baits. Genome assemblies were 658 obtained from the National Institute of Health's (NCBI) Genbank database.

\begin{tabular}{lcc}
\hline \multicolumn{1}{c}{ Taxon } & $\begin{array}{c}\text { BioProject } \\
\text { Accession }\end{array}$ & Reference \\
\hline Medusozoa: Hydrozoa: Hydra magnipapillata & PRJNA12876 & Chapman et al., 2010 \\
Medusozoa: Cubozoa: Alatina alata & PRJNA312373 & Ohdera et al., 2019 \\
Medusozoa: Staurozoa: Calvadosia cruxmelitensis & PRJEB23739 & Ohdera et al., 2019 \\
Anthozoa: Pentalucea: Renilla reniformis & PRJEB20462 & Ohdera et al., 2019 \\
Anthozoa: Scleractinia: Acropora digitifera & PRJDA67425 & Shinzato et al., 2011 \\
Anthozoa: Actiniaria: Nematostella vectensis & PRJNA12581 & Putnam et al., 2007 \\
\hline
\end{tabular}


660 Table 2 Species and sequence data sampled for phylogenetic analyses. Data for species in bold 661 were generated in this study. For each species, the number of loci and amino acid residues 662 included in the final concatenated 134 locus alignment are provided. NCBI: National Center for 663 Biotechnology Information; USNM: National Museum of Natural History, Smithsonian 664 Institution; UOGCVC: University of Guam Coral Voucher Collection. 


\begin{tabular}{|c|c|c|c|c|c|}
\hline & Taxon & Voucher/Reference & Loci & Residues & NCBI Accession \\
\hline \multicolumn{6}{|c|}{ H y d r o i d o l i n a } \\
\hline \multirow[t]{3}{*}{ Capitata } & Zancleopsis tentaculata & USNM1622168 & 12 & 1,103 & MW272249-60 \\
\hline & Millepora dichotoma & UOGCVC947 & 6 & 726 & MW272119-24 \\
\hline & Pennaria disticha & USNM1622068 & 47 & 4,403 & MW272261-307 \\
\hline Filifera I & Myrionema hargitti & USNM1622176 & 22 & 2,005 & MW272227-48 \\
\hline Filifera II & Proboscidactyla sp. & USNM1622170 & 5 & 448 & MW272435-39 \\
\hline \multirow[t]{7}{*}{ Leptothecata } & Dynanema crisioides & USNM1622069 & 62 & 6,173 & MW272032-93 \\
\hline & Nemalecium lighti & USNM1622067 & 54 & 5,256 & MW272125-78 \\
\hline & Aglaophenia parvula & USNM1621045 & 39 & 3,890 & MW272179-217 \\
\hline & Kirchenpaueria halecioides & USNM1622065 & 25 & 2,548 & MW272094-118 \\
\hline & Kirchenpaueria sp. & USNM1621044 & 84 & 8,018 & MW272308-91 \\
\hline & Octophialucium sp. & USNM1622151 & 40 & 3,334 & MW271992-031 \\
\hline & Clytia hemispherica & Kayal et al., 2018 & 26 & 4,266 & PRJEB32541 \\
\hline \multirow[t]{4}{*}{ Filifera III } & Podocoryne carnea & Kayal et al., 2018 & 109 & 16,709 & SRX529566 \\
\hline & Podocoryne martinicana & USNM1622132 & 9 & $\mathbf{1 , 0 7 7}$ & MW272218-26 \\
\hline & Hydractinia symbiolongicarpus & Kayal et al., 2018 & 104 & 16,411 & SRX474878 \\
\hline & Hydractinia polyclina & Kayal et al., 2018 & 107 & 16,950 & SRR923509 \\
\hline Filifera IV & Merga violacea & USNM1622162 & 10 & 831 & MW271982-91 \\
\hline \multirow[t]{6}{*}{ Siphonophorae } & Athorybia rosacea & USNM1622138 & 6 & 871 & MW272429-34 \\
\hline & Agalma elegans & Kayal et al., 2018 & 99 & 15,170 & SRX288285 \\
\hline & Nanomia bijuga & Kayal et al., 2018 & 96 & 14,339 & SRX288430 \\
\hline & Craseoa lathetica & Kayal et al., 2018 & 89 & 13,177 & SRX288432 \\
\hline & Abylobsis tetragona & Kayal et al., 2018 & 95 & 14,370 & SRX288276 \\
\hline & Physalia physalis & Kayal et al., 2018 & 105 & 15,455 & SRX288431 \\
\hline \multirow[t]{5}{*}{ Aplanulata } & Hydra magnipapillata ${ }^{1}$ & Kayal et al., 2018 & 94 & 14,171 & SRX000112 \\
\hline & Hydra magnipapillata ${ }^{2}$ & Chapman et al., 2011 & 105 & 16,187 & PRJNA12876 \\
\hline & Hydra oligactis & Kayal et al., 2018 & 64 & 8,342 & SRR040466-9 \\
\hline & Hydra viridissima & Kayal et al., 2018 & 70 & 9,237 & SRR040470-3 \\
\hline & Ectopleura larynx & Kayal et al., 2018 & 106 & 16,688 & SRX315375 \\
\hline \multicolumn{6}{|c|}{ O u t g r o u p } \\
\hline \multirow[t]{4}{*}{ Trachylina } & Craspedacusta sowerbii & Kayal et al., 2018 & 116 & 18,413 & SRR923472 \\
\hline & Aglaura hemistoma & USNM1622142 & 8 & 925 & MW271974-81 \\
\hline & Solmundaegina nematophora & USNM1284330 & 6 & 324 & MW271968-73 \\
\hline & Aegina citrea & Kayal et al., 2018 & 69 & 9,713 & SAMN03418514 \\
\hline \multirow[t]{2}{*}{ Cubozoa } & Alatina alata & Kayal et al., 2018 & 113 & 17,918 & SAMN03418513 \\
\hline & Tripedalia cystophora & Kayal et al., 2018 & 34 & 4,088 & $\begin{array}{l}\text { SRR1182852, } \\
\text { SRR2103559 }\end{array}$ \\
\hline \multirow[t]{7}{*}{ Scyphozoa } & Periphylla periphylla & Kayal et al., 2018 & 55 & 6,980 & SRR1915828 \\
\hline & Atolla vanhoeffeni & Kayal et al., 2018 & 85 & 12,468 & SAMN03418515 \\
\hline & Chrysaora chesapeakei & USNM1454941 & 37 & 3,145 & MW272392-428 \\
\hline & Cyanea capillata & Kayal et al., 2018 & 37 & 4,503 & SRR1930118 \\
\hline & Stomolophus meleagris & Kayal et al., 2018 & 103 & 15,551 & SRR1168418 \\
\hline & Cassiopea xamachana & Kayal et al., 2018 & 93 & 12,888 & $\begin{array}{l}\text { ERX2270394-7, } \\
\text { ERX2281610-3 }\end{array}$ \\
\hline & Aurelia aurita & Brekhman et al., 2015 & 111 & 17,342 & GBRG00000000 \\
\hline \multirow[t]{2}{*}{ Staurozoa } & Haliclystus auricula & USNM1621043 & 17 & 1,406 & MW271951-67 \\
\hline & Craterolophus convolvulus & Kayal et al., 2018 & 94 & 12,821 & ERR2248381 \\
\hline
\end{tabular}


667 Table 3 Log likelihood of maximum likelihood inferences under different topological constraints 668 (see Fig. 3). Likelihood ratios were calculated to compare alternate tree hypotheses to the null, $\mathrm{T}_{0}$ 669 (cf. Cartwright and Nawrocki, 2010). The approximately unbiased test (AU) was used to test the 670 null hypothesis that all tree topologies have the same log likelihood. Tree topology fit to the 671 alignment data was evaluated using the posterior probability of the Bayesian Information 672 Criterion (BIC).

\begin{tabular}{ccccc}
\hline Constraint & $\begin{array}{c}\text { Log } \\
\text { Likelihood }\end{array}$ & $\begin{array}{c}\text { Likelihood } \\
\text { Ratio }\end{array}$ & AU & $\begin{array}{c}\text { BIC } \\
\text { Posterior }\end{array}$ \\
\hline $\mathrm{T}_{0}$ & $-375,741.33$ & - & $\mathbf{0 . 5 4}$ & $\mathbf{0 . 2 8 2}$ \\
$\mathrm{T}_{1}$ & $-375,740.41$ & -0.92 & $\mathbf{0 . 6 0}$ & $\mathbf{0 . 7 1 8}$ \\
$\mathrm{T}_{2}$ & $-375,844.52$ & 103.19 & $2 \mathrm{e}-4$ & $4 \mathrm{e}-46$ \\
$\mathrm{~T}_{3}$ & $-375,944.68$ & 203.35 & $4 \mathrm{e}-4$ & $1 \mathrm{e}-89$ \\
\hline
\end{tabular}

673 
Figure 1

Summary of amino acid alignment completeness and taxon coverage.

A) Alignment of the 134 amino acid residue partitions; B) total number of amino acid residues contained in each alignment partition; C) number of species included in each alignment partition.

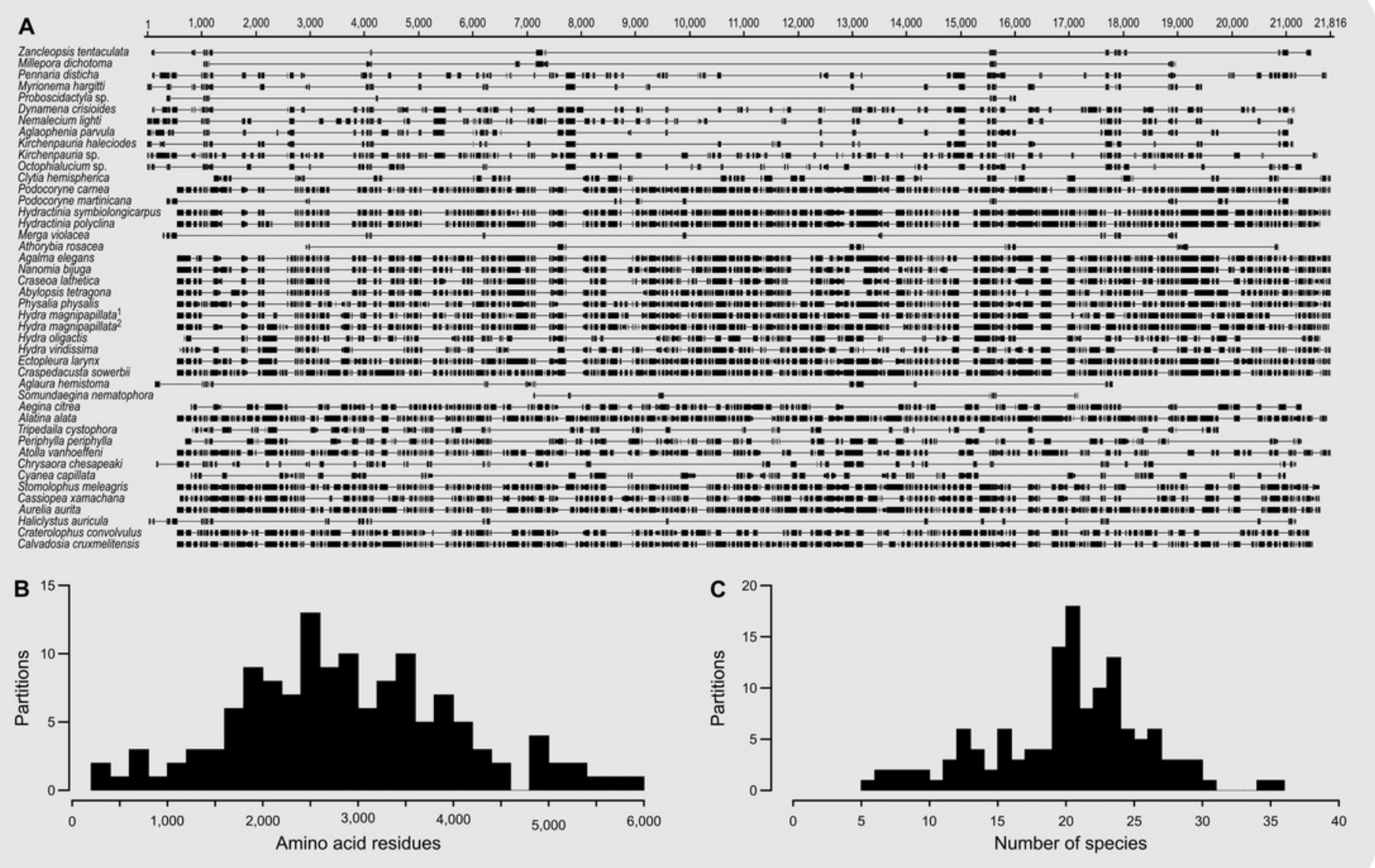




\section{Figure 2}

Cladogram of statistical gene tree summary derived from the most common quartet trees obtained from 134 bootstrapped gene trees.

Node labels represent mean quartet frequencies and give insight into areas of gene tree conflict in the dataset. Quartet frequencies of 1 indicate no gene tree discordance while lower frequencies indicate discordance (Mirabab, 2019).

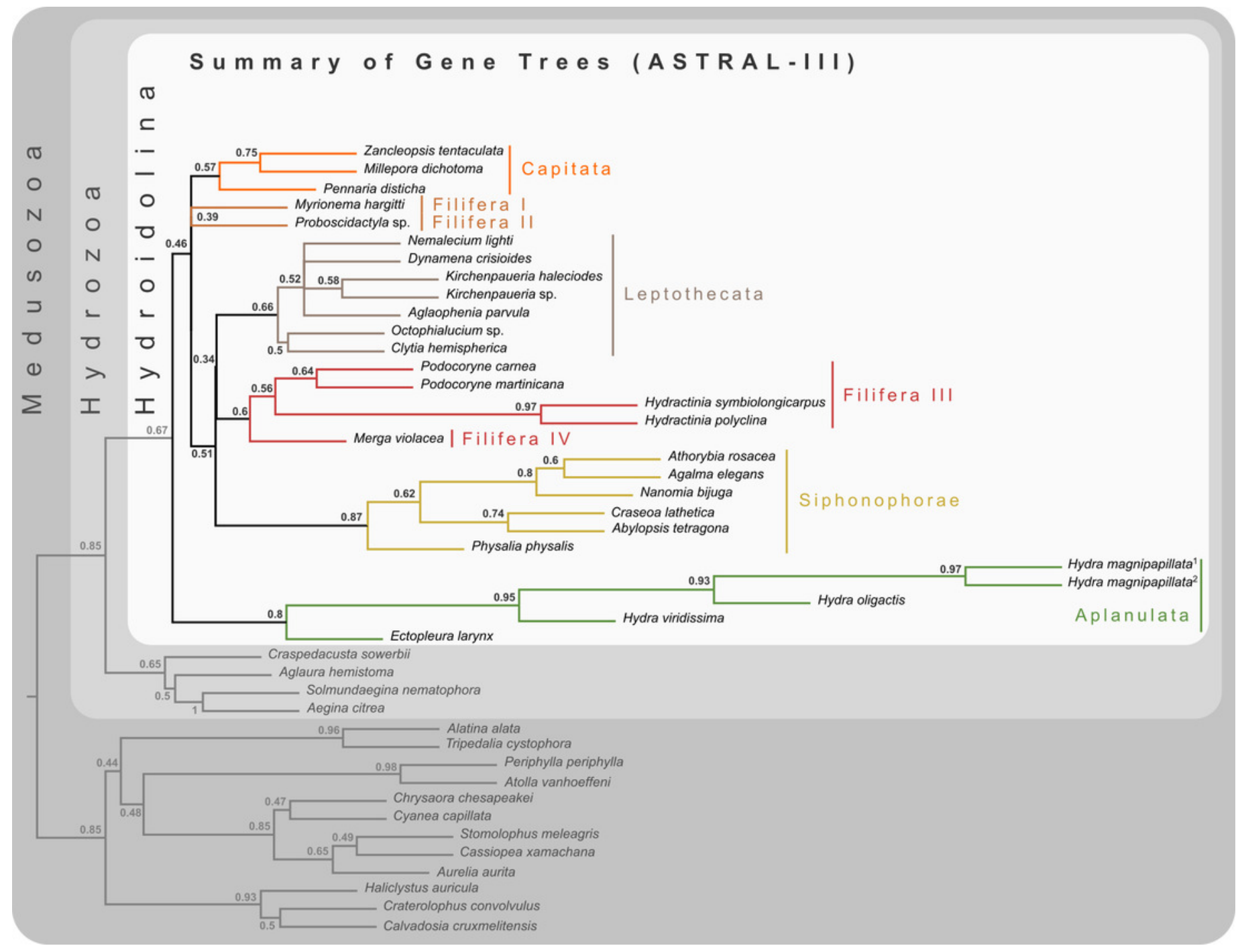




\section{Figure 3}

Bayesian and maximum likelihood phylogenies inferred from concatenated amino acid alignment.

Majority rule consensus of Bayesian phylogenetic inference under the CAT-GTR model (left side) with posterior probabilities given at each node. Maximum likelihood phylogeny (right side) was inferred from a partitioned analysis under the LG, WAG, and MtZoa models. Node support values represent the summary of 681 non-parametric maximum likelihood bootstrap searches. Scale bars indicate the number of substitutions per site. Scale bars indicate substitutions per site on each respective phylogram. 


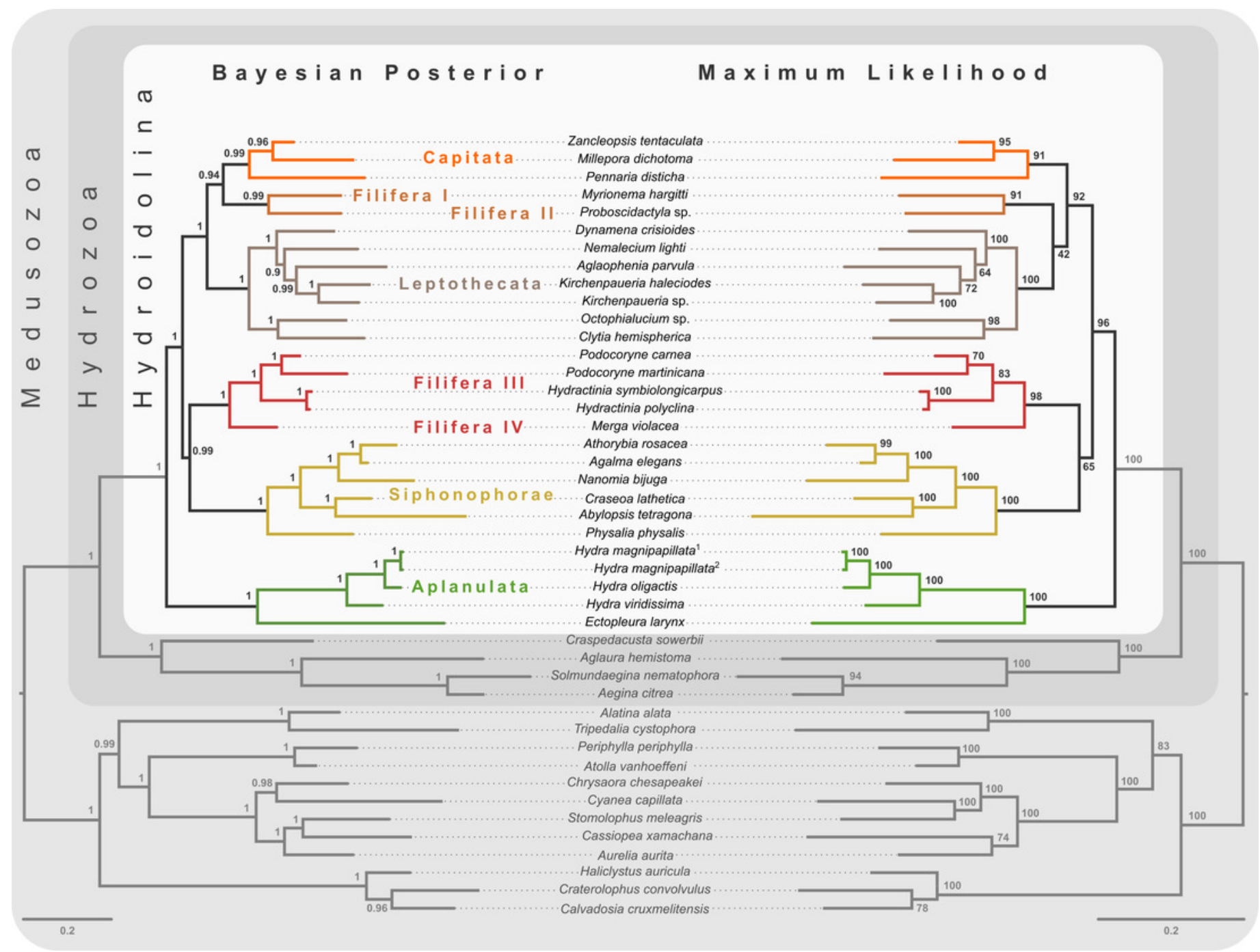




\section{Figure 4}

Minimally constrained maximum likelihood phylograms.

Constrained nodes are indicated by solid circles and the log likelihood for each phylogeny are given; scale bars indicates substitutions per site. The backbone of the phylogeny was constrained to maintain monophyly of the outgroup and monophyly of Hydroidolina with respect to Trachylina. Within Hydroidolina, three hypotheses of sister-group relationships were evaluated: Aplanulata (left), Capitata (center), or Siphonophorae (right) as sister to the remainder of Hydroidolina. The approximately unbiased test (AU) indicated that the Capitata (center) and Siphonophorae (right) sister-group hypotheses should be rejected. The Bayesian Information Criterion (BIC) showed a good fit of the Aplanulata (left) sister-group phylogeny to the alignment data.
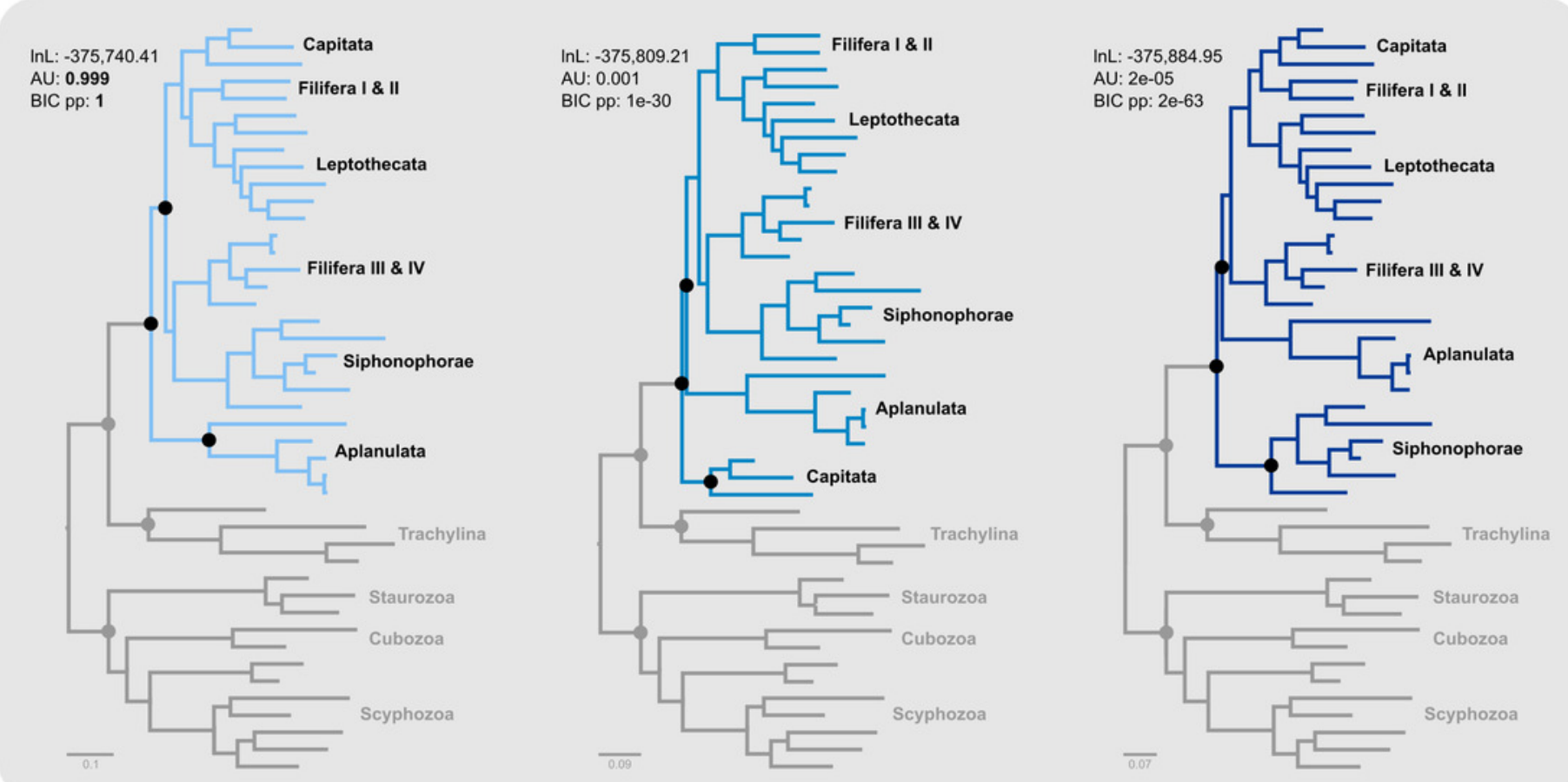


\section{Figure 5}

Phylogenetic hypothesis tests using fully constrained tree searches.

A) Phylograms inferred using fully constrained maximum likelihood searches to compare explicit hypotheses of tree topologies; constraints indicated by solid black circles; scale bar indicates substitutions per site. Tree topologies were constrained to reflect the Bayesian inference $\left(T_{0}\right)$ and maximum likelihood phylogenies $\left(T_{1}\right)$ presented in this contribution (Fig. 3). Two alternate tree searches were constrained to reflect previously published phylogenies $\left(T_{2}\right.$ : Cartwright \& Nawrocki, 2010; T3: Kayal et al., 2015); *** indicates a p-value of less than 0.001 for the approximately unbiased (AU) test (Table 3). Shallow nodes were collapsed and species labels removed for legibility. Scale bar indicates the number of substitutions per site. B) Each graph shows the log likelihoods of four independent Bayesian Markov Chain Monte Carlo (MCMC) runs following burn-in. Log likelihoods for both $T_{0}$ and $T_{1}$ were sampled from the posterior under the CAT-GTR and the CAT-Poisson (F81) model using the constraints shown in (A). C) Harmonic means representing the marginal likelihood (white circles) and log likelihood distributions of $T_{0}$ and $T_{1}$ under CAT-GTR (left) and CAT-Poisson (F81; right) models. Grey lines indicate the harmonic means for $T_{0}$. D) Bayes factors for pairwise comparisons between $T_{0}$ and $T_{1}(C)$. A negative Bayes factor provides evidence for $T_{0}$ while a positive Bayes factor indicates evidence against $T_{0}$, providing support for $T_{1}$. A Bayes factor between -2 and 2 indicates lack of evidence or no evidence favoring one hypothesis over the other, Bayes factors in the range of 2 to 6 ( -2 to -6$)$ provide some evidence, 6 to 10 (-6 to -10$)$ strong evidence, and Bayes factors $>10(<-10)$ very strong evidence for one hypothesis compared to the other. 
A
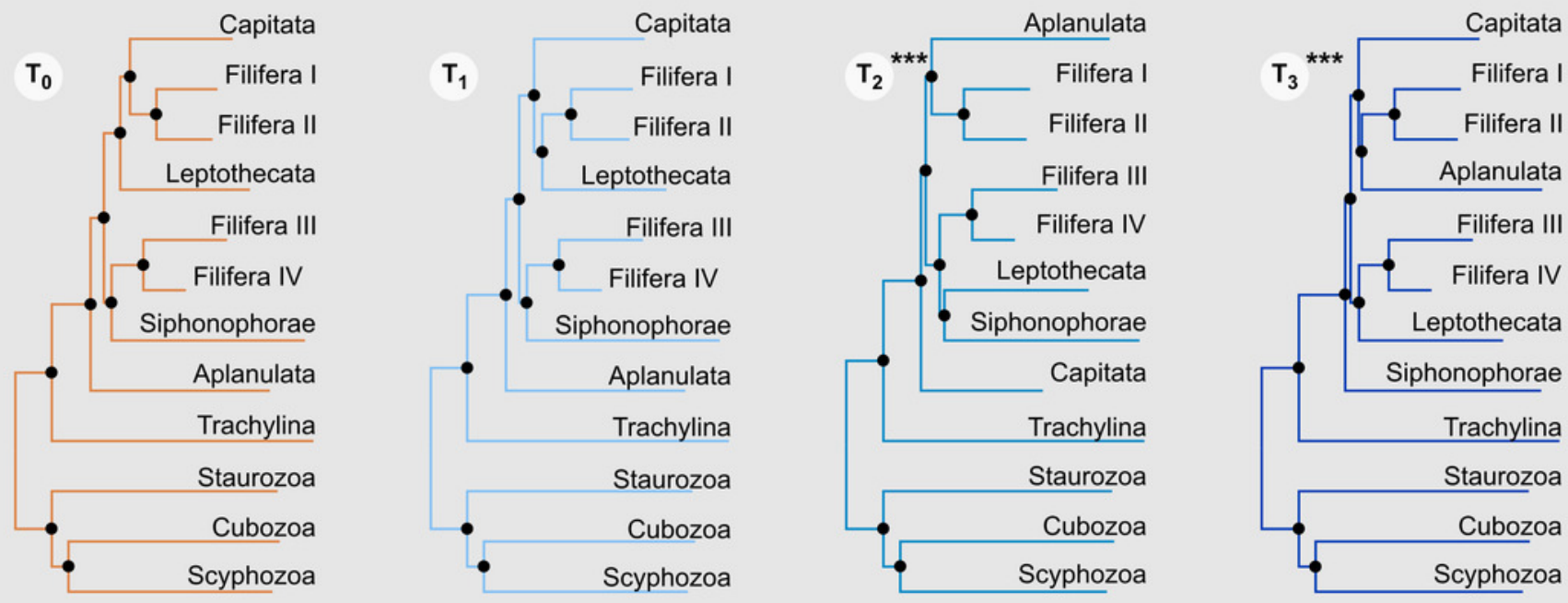

$\overline{0.1}$

B
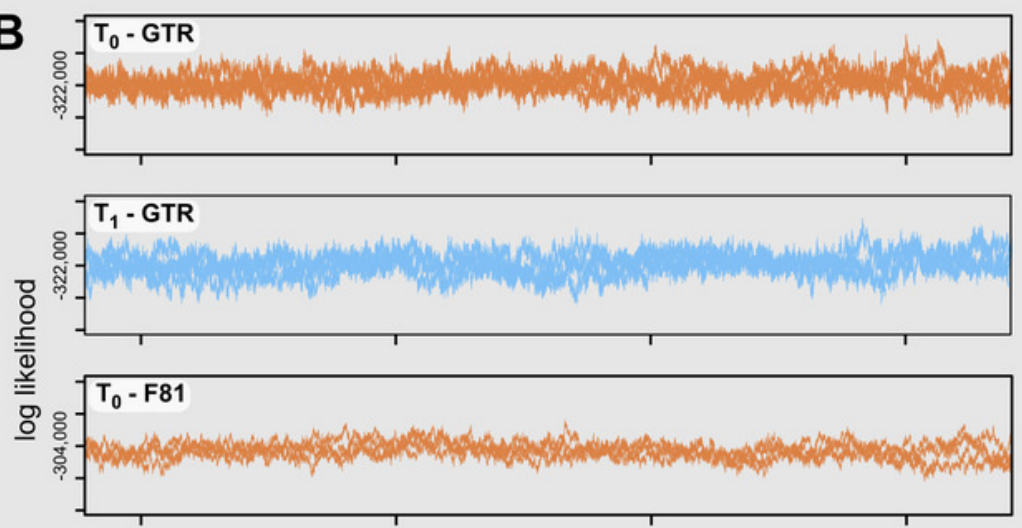

C
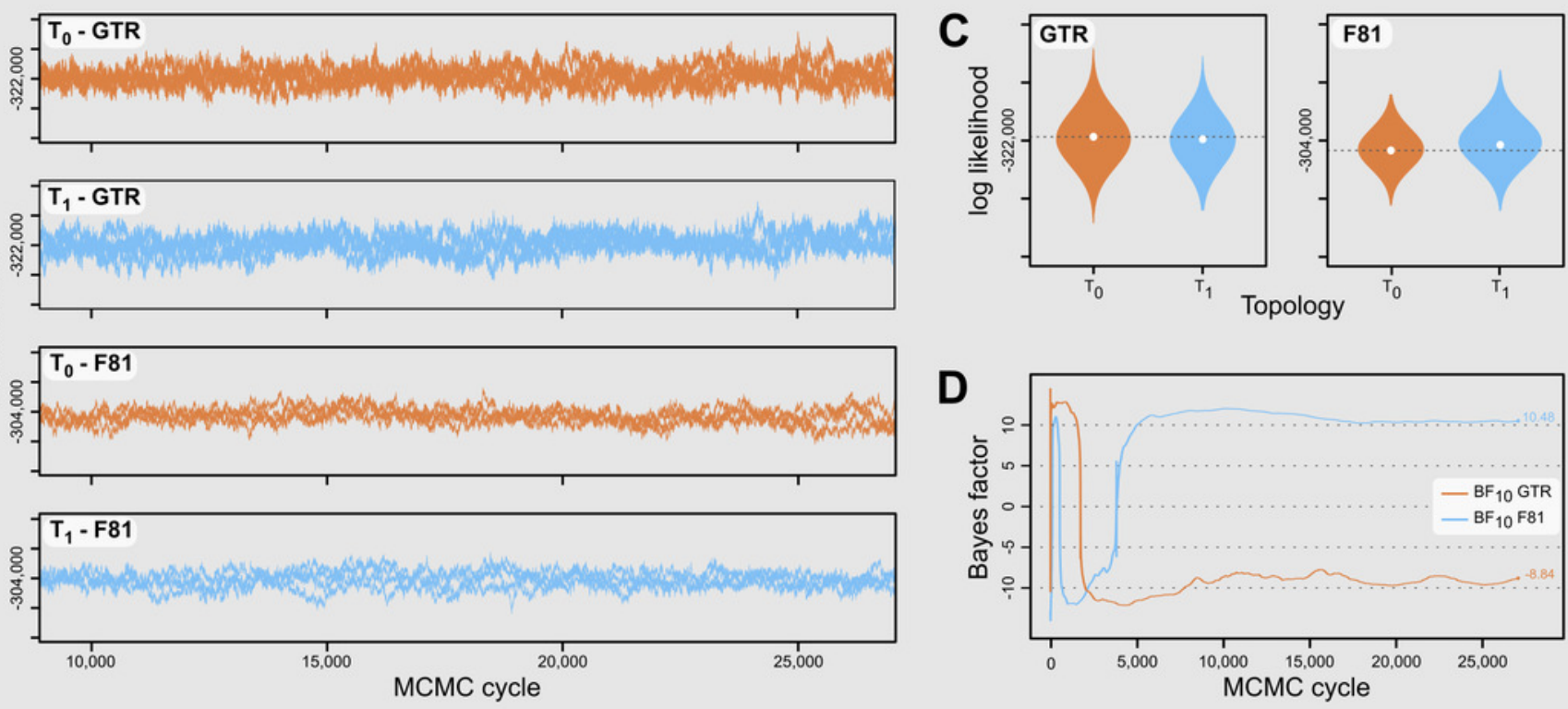